\title{
lon beam formation in a low-pressure geometrically expanding argon plasma
}

\author{
C. S. Corr, ${ }^{\text {a) }}$ J. Zanger, R. W. Boswell, and C. Charles \\ Space Plasma, Power, and Propulsion group, Research School of Physical Sciences and Engineering, \\ The Australian National University, Canberra, Australia Capital Territory 0200, Australia
}

(Received 7 September 2007; accepted 19 November 2007; published online 10 December 2007)

Supersonic ion beam formation has been observed in a geometrically expanding low-pressure
inductively coupled argon plasma. It is found that the ion beam is only observed below 3 mTorr and
only when the discharge is operated in inductive mode. The geometrical expansion of the plasma
induces density and potential gradients leading to the ion beam formation. The ion beam energy
increases with decreasing source tube radius. The results show that ion beam formation can be
achieved by geometrical expansion alone and that the ion beam energy depends on the ratio of the
cross-sectional area of the source and expansion region. (C) 2007 American Institute of Physics. [DOI: $10.1063 / 1.2823575$ ]

The process of plasma expansion and subsequent ion acceleration is of great interest to electric propulsion devices. In particular, low-powered miniature lightweight thrusters are currently of great interest to the propulsion community. Plasma expansion has been investigated experimentally, theoretically, and by computer simulation since the 1930s. ${ }^{1,2}$ In low-pressure isothermal plasmas, a pressure gradient $\nabla n T_{e}$ will produce a plasma pressure which will be balanced by an electric field. This Boltzmann field serves to reduce electron loss down the density gradient and to produce a drift in the ion distribution. The potential gradients generated by density gradients resulting from diverging magnetic fields, geometric expansion, or electric currents can induce supersonic flows of charged particles and may also result in the formation of a current free double layer. ${ }^{3-7}$ Ion sheaths in processing plasmas are essentially a single layer where the ion density is much larger than the electron density. Electric double layers are a two contiguous sheath phenomena, one sheath is composed of positive charges (ion sheath) and the second of negative charges (an electron sheath). ${ }^{8}$ A double layer, which is indicated by a sharp drop in plasma potential, can occur in low-pressure expanding plasmas and can occur naturally under certain operating conditions. This phenomenon is typically associated with astrophysical plasmas, but has recently been observed in a helicon discharge. Charles and Boswell ${ }^{3}$ investigated current-free double layers (DLs) occurring in an expanding helicon wave excited plasma discharge at very low pressures, typically less than 1 mTorr. The Chi Kung helicon system was operated with a strongly diverging static magnetic field, which was required to reach the conditions for DL formation and to obtain an ion beam with supersonic velocities. This type of thruster system has been named the helicon double layer thruster. This plasma system also has an abrupt change in radius at the boundary between the source and the diffusion chambers, which may also be a source of DL formation. When operated without a magnetic field, no DL or ion beam was observed in the Chi Kung system at low pressure. However, double layers and ion beam formation have been observed in expanding electronegative plasma discharges when operated without a magnetic field. ${ }^{9,10}$ The negative ions result in an alternative loss

\footnotetext{
${ }^{a)}$ Electronic mail: cormac.corr@anu.edu.au.
}

mechanism for electrons generating a double layer close to the source/diffusion chamber interface. Ion beam formation was also measured in the expansion region.

More recently, Shamrai et al. ${ }^{11}$ characterized a compact helicon source, which generated an ion beam at its exit. In particular they found that by optimizing operating parameters, the plasma density was raised up to $5 \times 10^{12} \mathrm{~cm}^{-3}$ and an ion beam of energy above $100 \mathrm{eV}$ was observed. In their case a magnetic field was required. Dunaevsky et al. ${ }^{12}$ have observed plasma acceleration in a capacitively coupled plasma discharge created in a capillary tube. They observed plasma flow with energies of several tens of eV. It was found that the potential gradient at the opening of the capillary and the high-temperature electrons in the capillary discharge induced the ion acceleration. In the two previous publications, a double layer was not observed.

In the present letter, we investigate ion beam formation in a low-pressure geometrically expanding plasma operating without a static diverging magnetic field and its dependence on the geometrical size of the plasma source.

The "Bilby" plasma reactor shown in Fig. 1 consists of a source tube with one end connected to a $14 \mathrm{~cm}$ diameter, $28 \mathrm{~cm}$ long aluminium diffusion chamber. Hence, the plasma expands into the diffusion chamber underneath the source region. At the other end of the source tube, a floating metal vacuum connection is attached and argon gas enters the system. The glass source tube is $20 \mathrm{~cm}$ long and $0.5 \mathrm{~cm}$ thick and can be 2, 4.5, or $6 \mathrm{~cm}$ in diameter. The air-cooled threeloop antenna is powered through a close-coupled pi-type

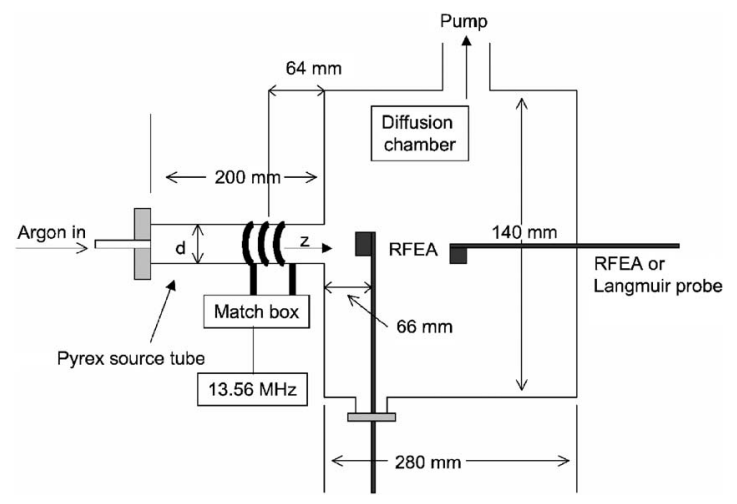

FIG. 1. Schematic of the inductively coupled Bilby reactor. 

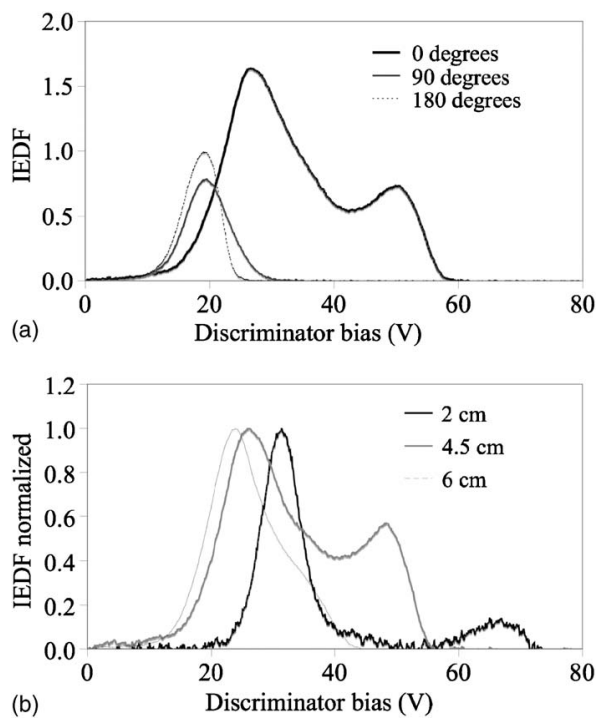

FIG. 2. (a) The ion energy distribution functions for an argon gas pressure of 0.9 mTorr and input power of $100 \mathrm{~W}$ for various RFEA orientations. (b) The ion energy distribution functions for an argon gas pressure of $1 \mathrm{mTorr}$ and input power of $100 \mathrm{~W}$ for various source diameters.

matching network by a rf power supply operating at 13.56 MHz and capable of delivering up to $500 \mathrm{~W}$ forward power. A pumping system consisting of a turbomolecular and a rotary pump are connected to the sidewall of the diffusion chamber and routinely achieve base pressures of $10^{-6} \mathrm{mTorr}$. The gas pressures are determined by controlling the flow of argon via a needle valve. Typical operating pressures were from 0.2 to 5 mTorr and were measured with an ionization gauge and convectron mounted on the diffusion chamber.

The plasma parameters were determined by electrostatic probe measurements. The plasma density, plasma and floating potential, and the electron temperature were determined from the $I(V)$ characteristics of a rf compensated Langmuir probe. A retarding field energy analyzer (RFEA) mounted on the side of the diffusion chamber $6.6 \mathrm{~cm}$ from the source/ diffusion chamber interface was used to determine the ion energy distribution function (IEDF). From the IEDF, one can determine the local plasma potential, the beam energy, and the beam and plasma density. The probe analysis here follows the methods described in Charles et al. ${ }^{13}$ and Plihon et al. ${ }^{10}$ RFEA's and Langmuir probes were also inserted through the diffusion chamber end opposite the source tube for axial measurements. Good agreement was found between the two methods.

Typical IEDF curves are shown in Fig. 2(a) for different angle orientations of the radial RFEA with respect to the source. The operating conditions are a source tube of $4.5 \mathrm{~cm}$, input power of $100 \mathrm{~W}$, and gas pressure of 0.9 mTorr. As can be seen from Fig. 2(a), two peaks are observed in the IEDF when the RFEA is facing the source. The first peak corresponds to the local plasma potential $\left(V_{p} \sim 25 \mathrm{~V}\right)$ while the second peak at higher energy is indicative of ion beam formation $\left(V_{\text {beam }} \sim 45 \mathrm{~V}\right)$. Ion beam detection in the diffusion chamber is confirmed when the RFEA is oriented to face away from the source. The higher energy second peak disappears above an angle of $40^{\circ}$, leaving only the lower energy peak at the local plasma potential. There is, however, a decrease in the plasma potential when changing the angular orientation of the RFEA. The reason for this is unclear, however, it should be noted that when the probe is facing the
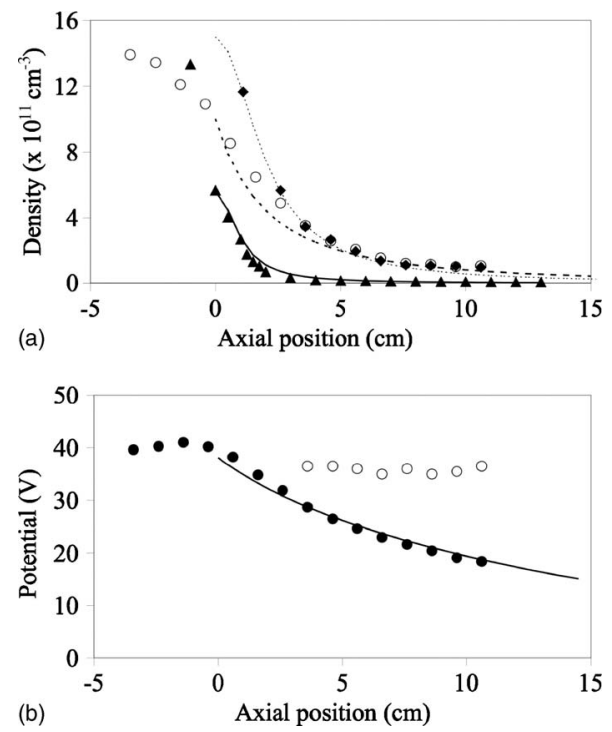

FIG. 3. (a) The measured plasma density for the $2 \mathrm{~cm}$ (triangles), $4.5 \mathrm{~cm}$ (solid diamonds), and $6 \mathrm{~cm}$ (open circles) source tubes. The lines correspond to the density calculated using Eq. (2); $2 \mathrm{~cm}$ (black line), $4.5 \mathrm{~cm}$ (dotted line), and $6 \mathrm{~cm}$ (dashed line). (b) The plasma (solid circles) and beam potential (open circles) as a function of axial position for the $6 \mathrm{~cm}$ source tube at $0.6 \mathrm{mTorr}$, and $100 \mathrm{~W}$. The line corresponds to $V_{p}$ calculated using Eq. (3).

source, the RFEA sees an uphill potential profile and when it faces away from the source it sees a downhill potential profile. This suggests that the change in plasma potential deduced from the RFEA may be related to the ion mean free path and the length of the presheath in front of the analyzer. The formation of the ion beam suggests that a DL may be formed in the region between the source and diffusion chamber. This is currently under investigation and will be the subject of a future publication.

IEDF curves are shown in Fig. 2(b) for the three source tubes used here, i.e., $2,4.5$, and $6 \mathrm{~cm}$ at a gas pressure of $1 \mathrm{mTorr}$ and an input power of $100 \mathrm{~W}$. The first observation to note is that two peaks are observed for all three configurations although for the $6 \mathrm{~cm}$ tube it is not as clear. The second point of interest is the change in the potential difference between the local ion energy and the ion beam energy indicating that a larger source-diffusion chamber radii ratio $\left(R_{\text {source }} / R_{\text {diff }}\right)$ leads to the formation of an ion beam with higher energy.

The increase in beam energy for a decreasing source radius is due to an increase in plasma density and potential gradients between the source region and the diffusion chamber. Figure 3(a) shows the axial variation of the plasma density for the three source tubes. The increase in the density gradient is clearly observed. The expanding spatial variation of the plasma density from the source into the diffusion chamber can be estimated in a similar way to that of a plasma jet, by taking the radius of the plasma expansion $R$ equal to

$$
\frac{R^{2}}{R_{0}^{2}}=1+\frac{z^{2}}{z_{0}^{2}},
$$

where $z$ is the $z$ coordinate, $R_{0}$ is the radius of the plasma source channel, and $z_{0}$ is the virtual source location. The location of the virtual source is set equal to the radius of the plasma source channel $R_{0}$ and is outside the plasma source channel. For $R_{0}=z_{0}$, expression (1) becomes $R=\sqrt{R_{0}^{2}+z^{2}}$ channel. For $R_{0}=z_{0}$, expression (1) becomes $R=\sqrt{R_{0}^{2}+z^{2}}$
o AlP license or copyright; see http://apl.aip.org/apl/copyright.jsp 

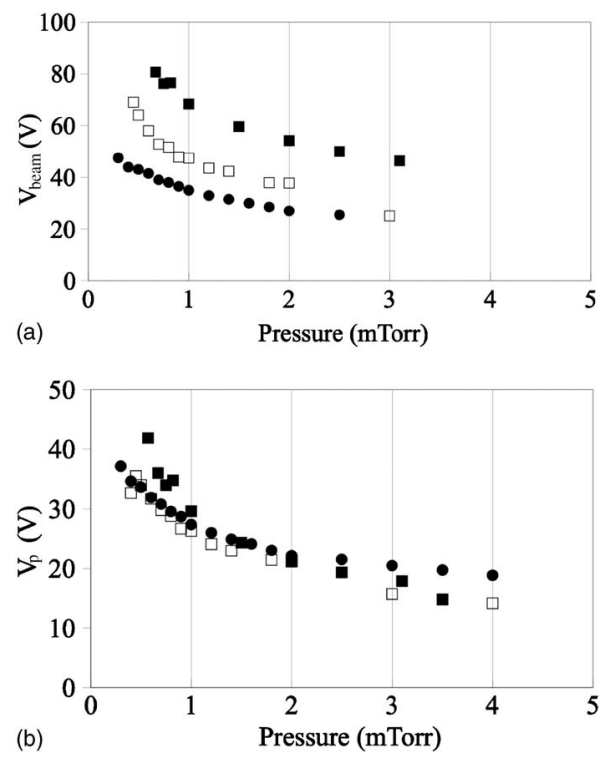

FIG. 4. The pressure dependence of (a) the beam energy and (b) the local plasma potential at $200 \mathrm{~W}$ for the $2 \mathrm{~cm}$ (squares), $4.5 \mathrm{~cm}$ (open squares), and the $6 \mathrm{~cm}$ (circles) source tubes.

which corresponds to a $45^{\circ}$ expanding plasma. This permits a continuous flow from the source tube into the expansion. From conservation of flow and expression (1), we obtain

$$
n(z)=n(0) \frac{R_{0}^{2}}{R_{0}^{2}+z^{2}},
$$

where $n(0)$ is the density at the source diffusion chamber interface, $R_{0}$ is the source tube radius, and $z$ is the axial distance from the interface. ${ }^{14}$ In Fig. 3(a), the lines show the calculated plasma density. An excellent agreement is observed between the model and experiment.

Figure 3(b) shows the axial variation of the plasma potential and beam potential for the $6 \mathrm{~cm}$ tube operated at $0.6 \mathrm{mTorr}$ and $100 \mathrm{~W}$. As can be seen from this figure, the beam clearly disappears on crossing the source tube/ diffusion chamber interface at $z=0 \mathrm{~cm}$. It should be noted that on approaching the interface it becomes more difficult to distinguish between the plasma potential and beam potential peaks. Although there is no sudden change in the plasma potential across the interface, it is still not possible to say if a double layer has not formed. This is due to the size of the RFEA, which perturbs the source plasma when entering the source tube. Assuming a constant Maxwellian electron distribution, the plasma density evolution is related to the plasma potential evolution by the Boltzman relation, ${ }^{15,16}$

$$
V_{p}(z)=V_{p}(0)+T_{e} \ln \left[\frac{n(z)}{n(0)}\right],
$$

where $T_{e}$ is the electron temperature $(8 \mathrm{eV})$ and $V_{p}(0)$ is the plasma potential at the source/diffusion chamber interface. From the density calculation of Eq. (1), the plasma potential was calculated. This is shown in Fig. 3(b) and again excellent agreement is observed.

Figures 4(a) and 4(b) show the evolution of the ion beam energy $\left(V_{\text {beam }}\right)$ and the local plasma potential $\left(V_{p}\right)$ as a function of pressure for the $2,4.5$, and $6 \mathrm{~cm}$ source tubes, respectively. The first observation is that the ion beam appears only at sufficiently low operating pressures. When increasing the pressure, both the local plasma potential and the ion beam energy decrease. The local plasma potential measured $6.6 \mathrm{~cm}$ from the source tube is of similar value for the three source tubes, however, at the lower pressure end, the plasma potential is higher for the smallest tube. When the pressure is increased above a threshold value, the second peak disappears. As the source diameter is decreased, there is an increase in the density and potential gradients leading to larger ion beam energies for all pressures investigated here.

Under the present conditions, the electron temperature has been measured with a compensated Langmuir probe to be $\sim 8 \mathrm{eV}$. The beam velocity and its Mach number can be estimated from

$$
v_{\text {beam }}=\sqrt{\frac{2 e\left(V_{\text {beam }}-V_{p}\right)}{M_{i}}}
$$

for each of the source tubes investigated here. It is found that at a pressure of $1 \mathrm{mTorr}$, the 2, 4.5, and $6 \mathrm{~cm}$ source tubes display beam velocities of $\sim 15.5, \sim 11.4$, and $\sim 6.9 \mathrm{~km} \mathrm{~s}^{-1}$, respectively. For all cases, the ion beams have velocities greater than the ion sound speed $c_{s} \sim 5 \mathrm{~km} \mathrm{~s}^{-1}$, i.e., all beams are supersonic.

In summary, we have shown that ion beam formation can occur at low pressures in a geometrically expanding compact plasma system without the use of an external magnetic field. We show that the supersonic ion beam is due to the geometrical expansion of the system with the beam acceleration occurring at the interface of the source tube and the diffusion chamber. Furthermore, varying the ratio of the cross-sectional areas of the source tube and the diffusion chamber can control the ion beam energy. It has been shown that the energy of the ion beam increases with decreasing source tube size. An experimental program is currently underway to determine if a double layer forms at the point of ion acceleration. This is difficult to determine in these small geometry reactors, as probe diagnostics is more intrusive. Although a large amount of input power is required to produce a small amount of thrust $(\mathrm{mN})$, this could be reduced by optimization of the rf antenna and the plasma cavity geometry. Such a simple and electrodeless thruster of this scale is a suitable candidate for electric propulsion in space such as in many modern small-scale satellites.

${ }^{1}$ G. Hairapetian and R. L. Stenzel, Phys. Fluids B 3, 899 (1991) and references therein.

${ }^{2}$ W. M. Manheimer and R. F. Fernsler, IEEE Trans. Plasma Sci. 29, 75 (2001).

${ }^{3}$ C. Charles and R. W. Boswell, Appl. Phys. Lett. 82, 1356 (2003).

${ }^{4}$ C. Charles and R. W. Boswell, Phys. Plasmas 11, 1706 (2004).

${ }^{5}$ C. Charles and R. W. Boswell, Phys. Plasmas 11, 3808 (2004).

${ }^{6}$ M. A. Lieberman and C. Charles, Phys. Rev. Lett. 97, 045003 (2006).

${ }^{7}$ M. A. Lieberman, C. Charles, and R. W. Boswell, J. Phys. D 39, 3294 (2006).

${ }^{8}$ C. Charles, Plasma Sources Sci. Technol. 16, R1 (2007).

${ }^{9}$ N. Plihon, C. S. Corr, and P. Chabert, Appl. Phys. Lett. 86, 091501 (2005).

${ }^{10}$ N. Plihon, P. Chabert, and C. S. Corr, Phys. Plasmas 14, 013506 (2007).

${ }^{11}$ K. P. Shamrai, Y. V. Virko, V. F. Virko, and A. I. Yakimeno, 42nd AIAA, 2006 (unpublished) p. 4845.

${ }^{12}$ A. Dunaevsky, Y. Raitses, and N. J. Fisch, Appl. Phys. Lett. 88, 251502 (2006).

${ }^{13}$ C. Charles, A. W. Degeling, T. E. Sheridan, J. H. Harris, M. A. Lieberman, and R. W. Boswell, Phys. Plasmas 7, 5232 (2000).

${ }^{14}$ K. T. A. L. Burn, W. J. Goedheer, and D. C. Schram, J. Appl. Phys. 90, 2162 (2001).

${ }^{15}$ C. Charles, R. W. Boswell, A. Bouchoule, C. Laure, and P. Ranson, J. Vac. Sci. Technol. A 9, 661 (1991).

${ }^{16}$ C. Charles, R. W. Boswell, and R. K. Porteous, J. Vac. Sci. Technol. A 10, 398 (1992). 